\title{
Impact of GBBS algorithm on post-mastectomy scar boost irradiation of breast using catheter flap
}

\author{
Mourougan Sinnatamby, PhD, Assist. Prof., Vijayaprabhu Neelakandan, PhD, Assoc. Prof., Gunaseelan Karunanidhi, MD, \\ Addit. Prof., Saravanan Kandasamy, PhD, Assist. Prof., Seenisamy Ramapandian, PhD, Assist. Prof., \\ Muniyappan Kannan, MSc, Elakiya Sampath, MSc \\ Department of Radiation Oncology, Regional Cancer Centre, Jawaharlal Institute of Postgraduate Medical Education and Research, \\ Puducherry, India
}

\begin{abstract}
Purpose: Post-mastectomy radiation therapy significantly reduces locoregional recurrence rates, which can be achieved with external beam radiotherapy delivered to chest wall, followed by scar irradiation either by electron or high-dose-rate (HDR) mould brachytherapy. The present study evaluates dosimetric advantage of Acuros ${ }^{\circledR}$ BV, a TG-186 MBDCA, over TG-43 formalism using ${ }^{192}$ Ir source for HDR brachytherapy in chest wall scar boost using catheter flap.

Material and methods: A total of 25 patients, free of cardiac and pulmonary co-morbidities, who met the inclusion criteria were involved in the study. Catheter flap made of silicon with 20 channels was used to deliver a total dose of 7.5 Gy/3 fx by HDR surface mould brachytherapy to delineated scar volume. Plan was optimized with iterative method to obtain desired results with TG-43 formalism, followed by Acuros ${ }^{\circledR}$ BV (GBBS algorithm) without altering dwell positions or time. The two algorithm plans were analyzed qualitatively and quantitatively with dose-volume histograms.

Results: The mean $\mathrm{D}_{98 \%}$ CTV-HDR_evl coverage decreased by $1.16 \%$ compared to TG-43, and near-maximum dose decreased by $8.18 \%(p=0.000)$, mean $\mathrm{D}_{\max }$ dose to CTV-HDR_evl, and mean $\mathrm{D}_{\text {mean }}$ dose was lesser by $6.25 \%(p=0.000)$ and $10.82 \%(p=0.000)$, respectively, compared to TG-43. Heart $\mathrm{D}_{2 \%}$ showed significant results, whereas $\mathrm{D}_{\text {median }}(\mathrm{cGy})$ revealed very significant difference. A $5 \mathrm{~mm}$ thick skin contour showed statistically significant results $(p=0.000)$ for $\mathrm{V}_{150 \%}$ and $\mathrm{V}_{200 \%}$.

Conclusions: The presented data showed how Acuros ${ }^{\circledR} \mathrm{BV}$, algorithm-based calculation in scar boost irradiation of breast, accounting for a mass density of the medium and scatter condition, considered actual dose prediction in a medium.

Key words: Acuros ${ }^{\circledR}$ BV, catheter flap, chest wall, scar boost, GBBS, surface mould.

\section{Purpose}

Post-mastectomy radiation therapy (PMRT) significantly reduces loco-regional recurrence (LRR) rates and improves overall survival in patients with high-risk breast cancer [1,2]. The dose regimen for PMRT is usually 50 Gy to the chest wall (CW), followed by an additional 10 Gy to the surgical chest wall scar, especially for patients with close or positive mastectomy margins and large (i.e., $\geq 5 \mathrm{~cm}$ ) tumors [3]. Radiation therapy in these cases is delivered by several modalities, including high-dose-rate (HDR) brachytherapy and external beam radiotherapy (EBRT). Among EBRT techniques, orthovoltage radiotherapy with beam energies in the range of $100-250 \mathrm{kV}$, or electrons or high energy photons can be used [4]. With the above-mentioned modalities, the most commonly applied techniques are electrons and surface mould HDR brachytherapy. Usually, (en)-facing electrons are used to deliver the boost dose. However, electron treatment planning and preparation are involved, as electron cut-outs and dose featherings require both the time and effort.

The treatment option is usually based on institutional resources and expertise, which results in local control, cosmesis, and toxicity compliance of the treatment. The most common practice for achieving this is tangential fields by 3D conformal radiotherapy, intensity-modulated radiotherapy (IMRT), or volumetric modulated arc therapy (VMAT), followed by (en)-face electron boost to the scar region. Post-operative scar boost radiation for the reconstructed breast presents many planning challenges due to the shape, size, and curvature of the scar [5]. The advantages of brachytherapy over electrons are a rapid dose fall-off outside the target volume and short treatment duration [6]. There is an extreme lack of studies investigating the role of HDR surface mould brachyther- 
apy scar boost following PMRT, in the setting of close or positive margins [7]. Our experience over many trials in designing the standard mould for scar boost irradiation and air gaps leads to inhomogeneity in dose. An attempt was made to use commercially available flaps for the treatment of chest wall boost. In contrast, boost treatment during adjunct radiotherapy, following breast conservation surgery is documented [8]; however, not much literature is available about effective chest wall boosts during PMRT.

A clinical trial is being conducted in our institute assessing local control and morbidity when treating post-operative chest wall in breast cancer using a combination of external beam radiotherapy and HDR surface mould brachytherapy with a catheter flap. This study proved an effective method of efficacy in dose coverage to tumor volume and sparing the underlying critical organs. In skin HDR brachytherapy, sources are located outside, in contact with the skin surface, or placed within a certain distance from the skin. Most treatment planning systems use the American Association of Physicist in Medicine (AAPM) Task Group (TG) 43 formalism, which is based on single-source dose superposition within an infinite water medium, without accounting for the true geometry, in which conditions for scattered radiation are altered by the presence of air [9].

Acuros ${ }^{\circledR}$ BV is a grid-based Boltzmann solver (GBBS), which directly resolves linear Boltzmann transport equation. It deterministically solves, what Monte Carlo codes solve stochastically. Acuros ${ }^{\circledR} \mathrm{BV}$ in BrachyVision version 10.0 reported dose through medium but absorbed dose to water. Absorbed dose to the material was added from version 13.0 onwards. The purpose of this study was to evaluate the dosimetric advantage of using Acuros ${ }^{\circledR} \mathrm{BV}$ dose calculation algorithm with ${ }^{192}$ Ir source for HDR brachytherapy in breast scar boost treatment using catheter flap over TG-43 dose calculations.

\section{Material and methods}

\section{Patients' selection}

Patients required a PMRT boost to their chest wall who reported to have close or positive surgical margins, and were free of cardiac and pulmonary co-morbidities were included in this study. Total of 25 patients who met these inclusion criteria were involved in the study. Left-sided breast $(n=11)$ and right-sided breast $(n=14)$ patients were planned for external beam radiotherapy with scar boost irradiation using HDR surface mould brachytherapy as a sandwich.

\section{Inclusion and exclusion criteria}

Inclusion criteria were as follows: locally advanced carcinoma breast patients (IIIA, IIIB, and IIIC stages), histopathology of infiltrating ductal carcinoma, age $<60$ years, ECOG performance score 0 and 1, no chest wall irradiation in the past, no previous history of any cardiac disease and pulmonary dysfunction.

Exclusion criteria were as follows: age $>60$ years, ECOG performance score 2 and 3, history of previous radiation to the chest wall, and previous history of any cardiac disease and pulmonary dysfunction.

\section{Catheter flap}

Catheter flap ${ }^{\circledR}$ is commercially available (Varian Medical Systems, Palo Alto, CA, USA) device, which measures $200 \mathrm{~mm} \times 290 \mathrm{~mm} \times 10 \mathrm{~mm}$, made of silicon with 20 treatment channels. The mould probe measures $1.8 \mathrm{~mm}$ diameter $\times 320 \mathrm{~mm}$, with made of stainless steel and PA (polyamide-nylon) mandarin. Each channel can be identified with the help of channel marker clips. If required, the catheter flap can be cut to the necessary size, and is used for treating superficial cancers (scar boost irradiation), and the function of the flap is to create a space between the skin and source. The catheters are placed in the middle of the axis flap and spaced $5 \mathrm{~mm}$ apart. The probes ensure that the source is placed in a planned position with high precision. Before the catheter flap placement onto the patient skin, biocompatible sterile pads can be placed on patients' treated area to avoid direct contact with the skin. This sterile pad is less than $1.0 \mathrm{~mm}$, has a minor dosimetric impact, and can be accounted for a bolus to avoid contamination and cross-infection.

\section{Pre-treatment planning}

In this study, the patient's treatment position was maintained in EBRT and surface mould HDR brachytherapy, and documented. Thermoplastic masks were used to immobilize the patient who was placed in a supine position on a carbon fibre breast board, with an ipsilateral arm abducted above the head. This position would help image registration and reproducibility, as a catheter flap for surface mould brachytherapy for scar boost was placed on the chest wall under thermoplastic cast, as shown in Figure 1. Separate planning computed tomography (CT) for EBRT and surface mould brachytherapy was performed before the start of EBRT. Computed tomography simulation using Siemens Somatom Spirit equipment, with $3 \mathrm{~mm}$ slice thickness and $1 \mathrm{~mm}$ reconstructed image for HDR brachytherapy was performed for every patient.

\section{Target delineation and treatment regime}

The contour for all the patients in this study was delineated based on the Radiation Therapy Oncology Group (RTOG) criteria. For HDR surface mould brachytherapy using catheter flap, clinical target volume (CTV) was drawn using radio-opaque $(1 \mathrm{~mm}$ lead wire) markers placed on the scar, with $3 \mathrm{~cm}$ in lateral extension and $3 \mathrm{~cm}$ in craniocaudal direction of the scar. Since lead wire can cause attenuation and account for dose difference in Acuros ${ }^{\circledR}$ BV calculation, the Hounsfield units (HU) value for the lead wire was assigned to air equivalent, and were removed during the treatment. A $5 \mathrm{~mm}$ thick uniform structure lying beneath the skin, excluding $5 \mathrm{~mm}$ from the skin surface, was delineated with boundaries limited to wire markers. A $5 \mathrm{~mm}$ thick skin structure was created over the CTV-HDR_evl to restrict the dose constraints during optimization. A dose of 50 Gy in $25 \mathrm{fx}$ (2 Gy/fx) to the chest wall (PMRT) and for supracla- 

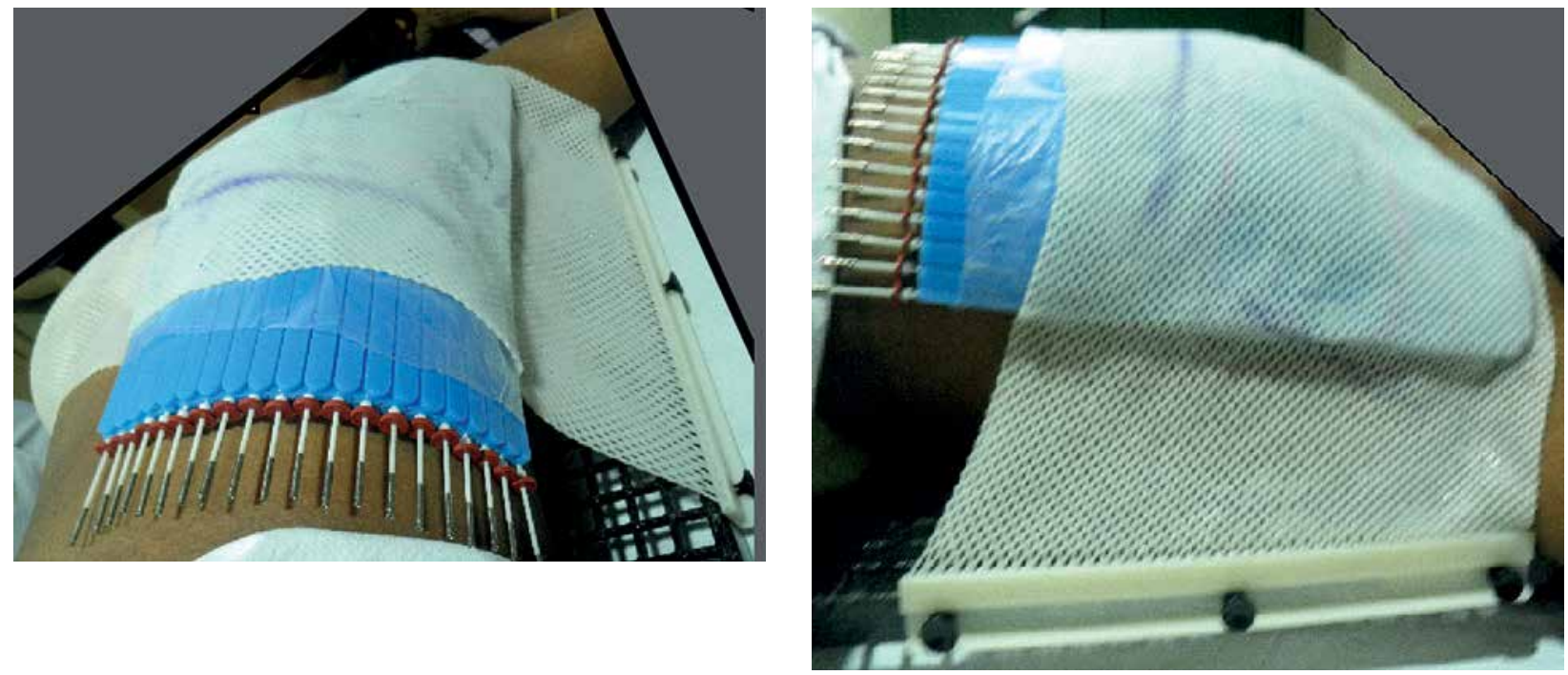

Fig. 1. Position of catheter flap with thermoplastic mask during simulation

vicular lymph node (SCLN) with axillary level of three nodes, and 7.5 Gy/3 fx (2.5 Gy/fx) was delivered by HDR surface mould brachytherapy to the delineated scar volume. The first fraction of HDR brachytherapy was given after seven fractions of EBRT, the second fraction after 14 fractions of EBRT, and the third fraction of HDR surface mould was applied after 21 fractions of EBRT. During the brachytherapy schedule, no EBRT treatment was applied.

\section{Treatment planning algorithms and optimization}

All the patients were contoured, planned, and evaluated by a single evaluator to minimize interpersonal variations. All plans were optimized and calculated using Varian BrachyVision (version 13.0), which has both standard TG-43 formalism, and Acuros ${ }^{\circledR}$ BV (version 1.4.0) developed by Transpire, Inc., Gig Harbor, Washington, USA, which was integrated into Eclipse treatment planning system (Varian Medical Systems Inc., Palo Alto CA, USA). Planned treatments were executed in GammaMedPlus ${ }^{\circledR} \mathrm{iX}$ (Varian Medical Systems Inc., Palo Alto CA, USA), with a maximum activity of $370 \mathrm{GBq}{ }^{192} \mathrm{Ir}$ stepping radioactive source to deliver the prescribed dose with decay correction. HDR brachytherapy plans were firstly performed using TG-43 formalism and inverse planning adaptive volume optimization. This was achieved with specific objectives for target volume and normal tissue sparing, followed by GBBS algorithm-based calculation (Acuros ${ }^{\circledR}$ BV). During planning, surface mould brachytherapy with catheter flap calculation resolution was set to $1 \mathrm{~mm}$ as a standard to provide the best output. Similarly, the matrix grid was identically ensured in both the calculation methods. Both EBRT and HDR surface mould brachytherapy plans were evaluated at the same time in order to arrive at a conclusion on organ dose-limiting and to evaluate skin dose.

\section{Plan evaluation and analysis}

All the planning was optimized using an iterative method to obtain desired results with TG-43 formalism.
If not achieved, isodose re-shaper tools were used at a particular location to improve the dose coverage. Once the plan was generated, the Acuros ${ }^{\circledR}$ BV algorithm was used without altering the dwell position used in the TG-43 calculation. However, the GBBS algorithm does take into account mass density information for computing dose. Dose distribution was compared between two algorithm plans and analyzed qualitatively and quantitatively with dose-volume histograms (DVHs).

Based on cumulative DVH and differential DVH, the following parameters for target and OARs were obtained:

1. $\mathrm{D}_{\min }, \mathrm{D}_{\max }, \mathrm{D}_{\text {mean }}, \mathrm{D}_{2 \%}, \mathrm{D}_{98 \%}, \mathrm{D}_{90 \%}, \mathrm{~V}_{100 \%}, \mathrm{~V}_{150 \%}$ for CTV-HDR_evl;

2. $\mathrm{D}_{\max }$ doses for contralateral breast, contralateral lung, ipsilateral lung, ribs, and skin;

3. $\mathrm{D}_{\text {mean }}$ doses for contralateral breast, heart, contralateral lung, ipsilateral lung, ribs, and skin;

4. $\mathrm{D}_{2 \%}$ doses for heart and ribs;

5. $\mathrm{V}_{5 \%}$ and $\mathrm{V}_{10 \%}$ for contralateral breast;

6. $\mathrm{V}_{150 \%}$ and $\mathrm{V}_{200 \%}$ for skin (volume receiving $150 \%$ and $200 \%$ of dose in skin structure, which were $5 \mathrm{~mm}$ above CTV-HDR_evl).

Calculations were also performed for volumetric homogeneity parameters, including:

- coverage index (CI): target volume ref/target volume, where target volume ref is the volume of the target that receives the reference dose;

- dose homogeneity index (DHI): $1-\mathrm{V}_{1.5}$ ref $/$ target volume ref, where $\mathrm{V}_{1.5}$ ref is the target volume that receives a dose of 1.5 times the reference dose;

- overdose volume index (OI): $\mathrm{V}_{2.0}$ ref $/$ target volume ref, where $V_{2.0}$ ref is the target volume that receives a dose of 2.0 times the reference dose;

- dose non-uniformity ratio (DNR): $\mathrm{V}_{1.5}$ ref $/$ target volume ref.

The statistical analysis of mean, standard deviation, 95\% CI of mean, and quartile deviation were applied, and plots were performed using IBM SPSS 21 software. Pair $t$ parametric tests were performed comparing two groups of data. 


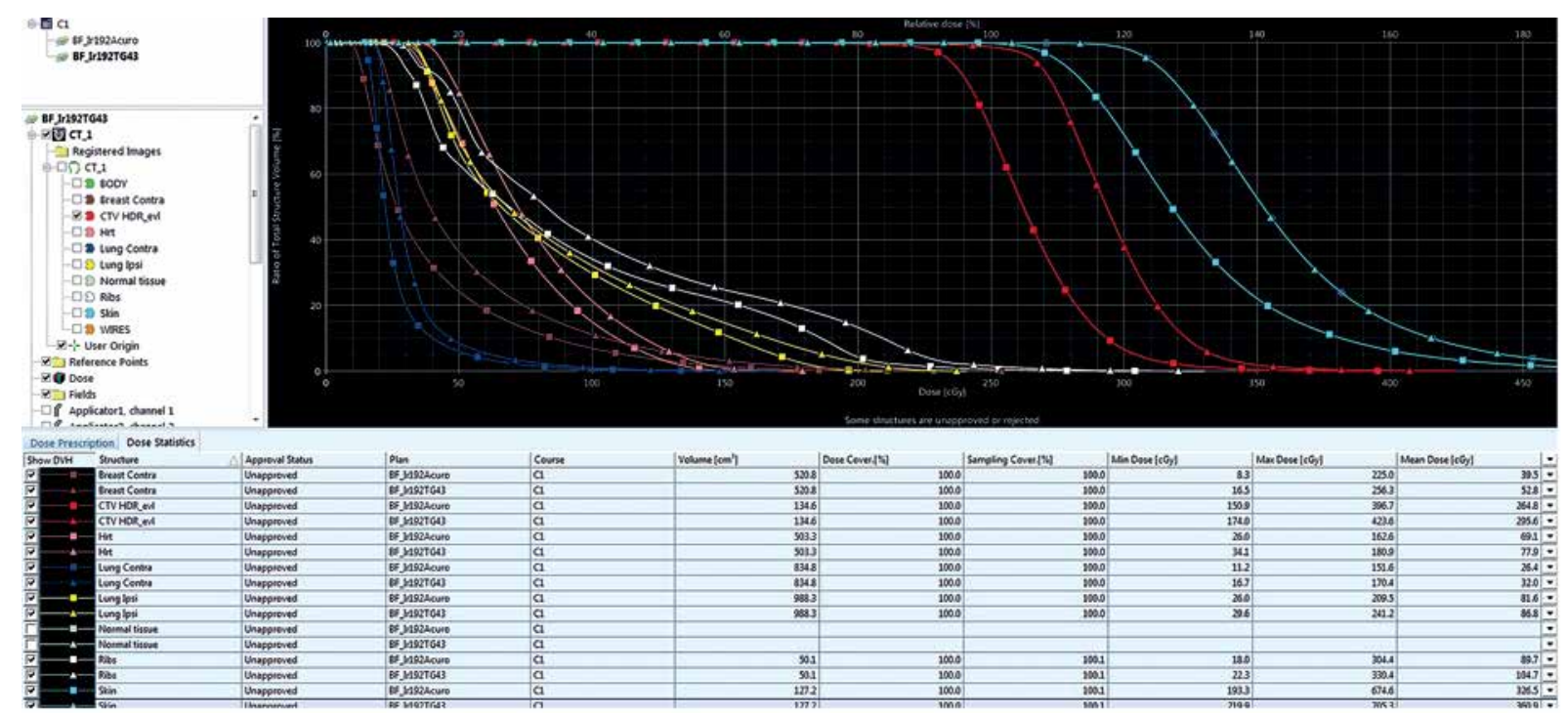

Fig. 2. Dose-volume histogram comparing TG-43 and Acuros ${ }^{\circledR}$ BV-based planning algorithm for clinical target volume (CTV) and organs at risk (OARs) in chest wall scar boost irradiation using catheter flap

\section{Results}

The CTV-HDR_evl volume ranged from 48 cubic centimeters (cc) to $186.4 \mathrm{cc}$. The number of channels required for the treatment of different volumes of CTV ranged from 10 to 20 . Figure 2 shows dose distribution for post-mastectomy scar boost irradiation comparing both TG-43 and Acuros ${ }^{\circledR}$ BV plans. The dose-comparison and its corresponding significance for different parameters in CTV are shown in Table 1 . The mean dose for $D_{98 \%}$ of CTV-HDR_evl for Acuros ${ }^{\circledR}$ BV was $88.19 \%$ (SD = 6.6) compared to $96.25 \%\left(\mathrm{SD}=7.4\right.$ ) for TG-43. The $\mathrm{D}_{2 \%}$ for CTV showed $138.5 \%(S D=12.4)$ for $\mathrm{TG}-43$ vs. $128.0 \%(\mathrm{SD}=11.8)$ with Acuros ${ }^{\circledR}$ BV. The mean $\mathrm{D}_{98 \%}$ CTV-HDR_evl coverage decreased by $10.45 \%(p=0.000)$ and near-maximum dose reduced by $7.56 \%(p=0.000)$ for Acuros ${ }^{\circledR} \mathrm{BV}$ plan as compared to TG-43-based calculation. The mean $D_{\max }$ dose to CTV-HDR_evl and the mean $D_{\text {mean }}$ dose were lesser by $5.85 \%(p=0.000)$ and $9.76 \%(p=0.000)$, respectively, as compared to TG-43. The dose homogeneity index and dose non-uniformity ratio indicated statistically significant results. However, overdose-volume index presented findings that were close to statistical significance.

In the contralateral breast, the mean $\mathrm{V}_{5 \%}$ showed a decrease in the dose by $33.76 \%$, and the mean $\mathrm{V}_{10 \%}$ indicated a reduction of $39.35 \%(p=0.000)$ with respect to TG-43based calculation. The mean $\mathrm{V}_{5 \%}$ and $\mathrm{V}_{10 \%}$ showed very significant differences between the two algorithms, but the maximum dose demonstrated close to statistically significant results $(p=0.04)$. The heart $\mathrm{D}_{2 \%}(\mathrm{cGy})$ showed a significant outcome, with a decrease in the dose by $5.73 \%$, whereas $\mathrm{D}_{\text {median }}$ (cGy) presented a very significant difference, with a reduced dose by $9.49 \%$ vs. TG- 43 . The mean dose to the heart with Acuros ${ }^{\circledR}$ BV plan was 81.6 cGy compared to 91.6 cGy with TG-43. In the contralateral lung, $D_{\text {mean }}, D_{\text {max }}$ and $V_{5 \%}$ showed very significant differences compared to Acuros ${ }^{\circledR}$ BV-based dose computation. In this study, the ribs of the diseased side were included for comparison, of which $\mathrm{D}_{2 \%}$ indicat- ed substantial results. The near maximum dose to the ipsilateral ribs with Acuros ${ }^{\circledR}$ BV plan was 198.36 cGy $(\mathrm{SD}=51.94)$ compared to $227.82 \mathrm{cGy}(\mathrm{SD}=55.68)$. A $5 \mathrm{~mm}$ thick skin contour was delineated over the CTV-HDR_evl, and the dose difference between the two algorithms showed statistically very significant results $(p=0.000)$ for $\mathrm{V}_{150 \%}$ and $\mathrm{V}_{200 \%}$.

In descriptive statistics, the box and whisker plot explained the minimum value, first quartile, median, third quartile, and maximum value, distribution, and skewness of the data. In Figure 3, two algorithms were compared to show respective medians, interquartile ranges, dispersion, overall spread, and signs of skewness, with potential outliers for max doses, mean doses, and nearmax doses of OARs. Since the skin dose was significant, $\mathrm{V}_{150 \%}$ and $\mathrm{V}_{200 \%}$ were also compared between TG-43 and Acuros ${ }^{\circledR}$ BV-based calculation. It was evident that all OARs, $D_{\text {mean }}$ and $D_{\max }$ values were relatively lower in doses compared to TG-43 calculation. Similarly, $\mathrm{D}_{\min }$ doses also followed the same pattern of results, and a relative reduction in dose was seen with Acuros ${ }^{\circledR}$ BV-based planning. The mean dose plot demonstrated positive skew for the ribs and negative skew for the ipsilateral lungs. The $\mathrm{D}_{2 \%}$ of all OARs indicated, the heart was lower in the dose compared to the ipsilateral ribs with TG-43. When Acuros ${ }^{\circledR}$ BV was used, the dose reduction was observed due to tissue inhomogeneity, which predicted more accurate dose computation. The $\mathrm{D}_{2 \%}$ for the ipsilateral lungs was negatively skewed. The $\mathrm{V}_{150 \%}$ and $\mathrm{V}_{200 \%}$ of the skin indicated positively skewed data for Acuros ${ }^{\circledR}$ BV.

\section{Discussion}

In this study, a comparative analysis was carried out to investigate the significance of heterogeneity algorithm in HDR surface mould brachytherapy for chest wall scar boost irradiation using traditional AAPM TG-43 formalism. The vast majority of patients experience isolated 


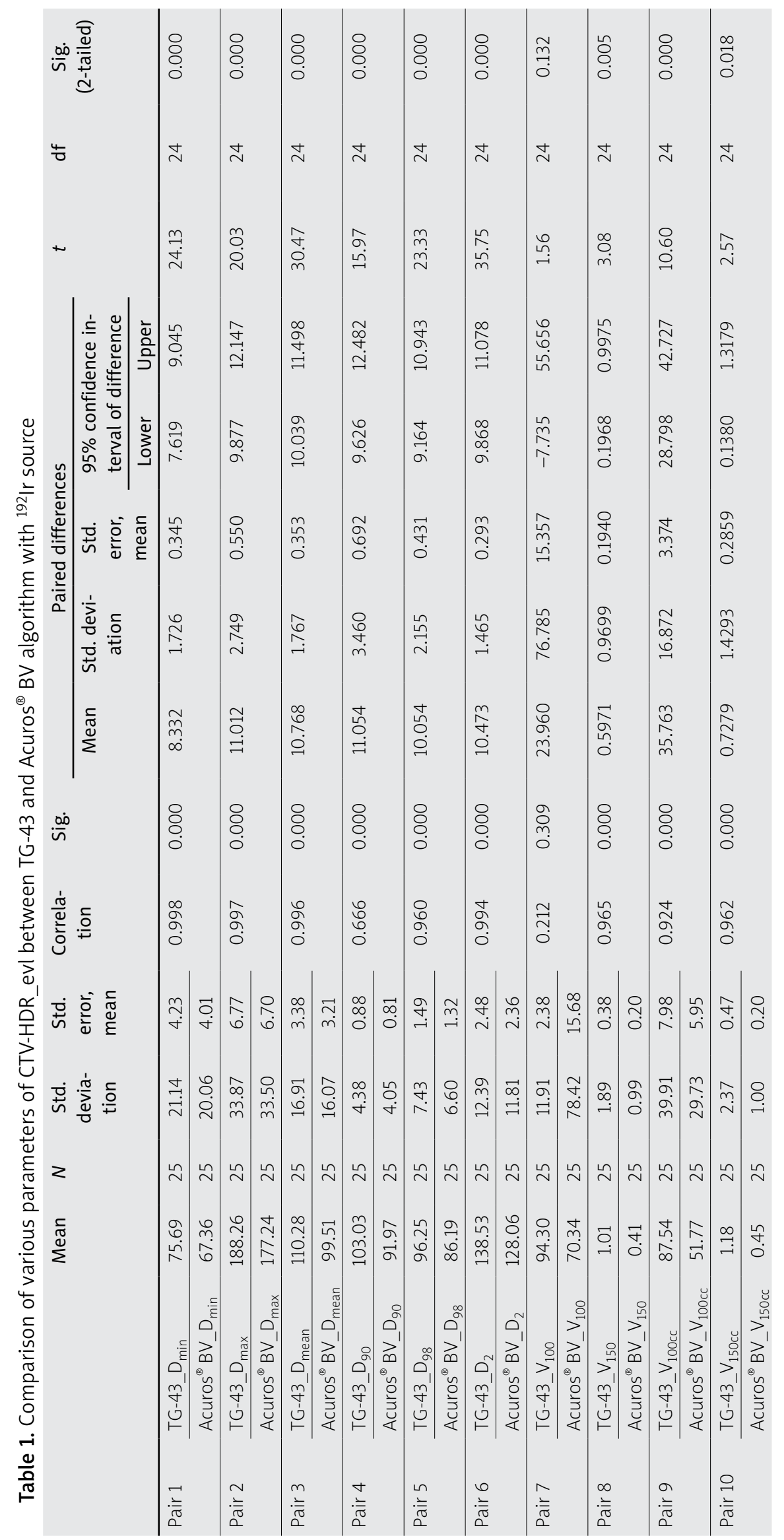


A

Comparison of max doses with TG-43 and Acuros $^{\circledR}$ BV algorithm

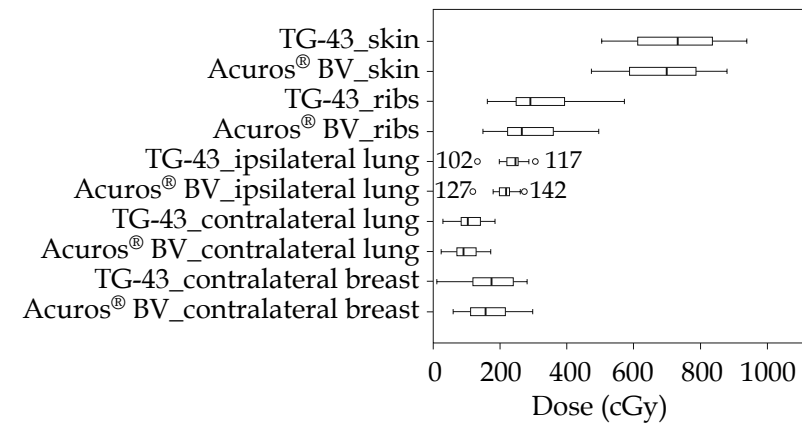

C Comparison of $\mathrm{D}_{2 \%}$ of volume of OARs with TG-43 and Acuros ${ }^{\circledR} \mathrm{BV}$ algorithm

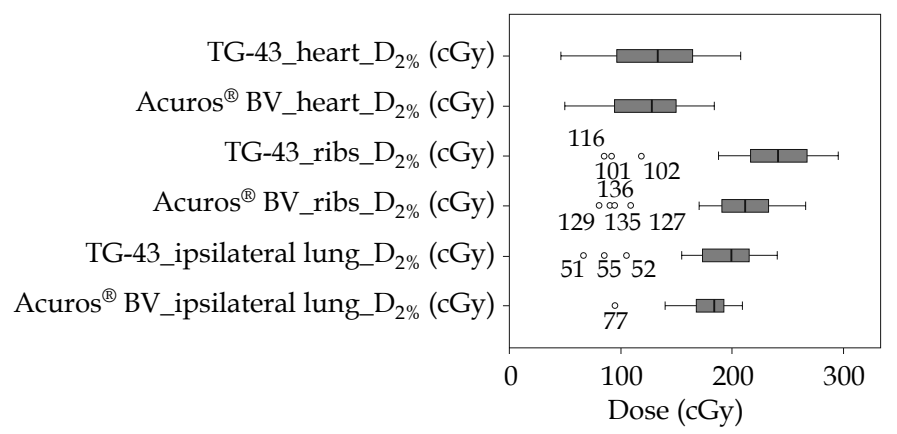

Fig. 3. The box and whisker plot. A) Comparison of max doses of organs at risk (OARs) with TG-43 vs. Acuros ${ }^{\circledR}$ BV algorithm-based planning in post-mastectomy scar boost irradiation. B) Comparison of mean doses of OARs with TG-43 vs. Acu$\operatorname{ros}^{\circledR} B V$ algorithm. C) Comparison of $D_{2 \%}$ of heart, ribs, and ipsilateral lung between TG-43 vs. Acuros ${ }^{\circledR} B V$ algorithm. D) $V_{150 \%}$ and $V_{200 \%}$ volume of skin were compared between TG-43 and Acuros ${ }^{\circledR}$ BV algorithm

chest wall failure and later develop distant metastasis within five years of loco-regional recurrence, with $25 \%$ chance of a recurrence after mastectomy for T1-2 N0 disease [10]. The catheter flap application to boost the chest wall scar provides a more conformal and homogeneous dose coverage for the scar volume compared to the electron field. Because the applicator curves with the chest wall can provide a homogeneous dose to the skin surface and $0.5 \mathrm{~cm}$ depth, it also avoids the need to use two or more matching electron fields [11]. If HDR surface mould brachytherapy is used for surgical scar boost as post-mastectomy radiotherapy, then accounting for an accurate dose prediction is possible with Acuros ${ }^{\circledR}$ BV algorithm. The catheter flap for scar boost irradiation is enclosed by immobilization cast, which ensures reproducibility over fractions and minimizes air gaps between skin and catheter flap. Sandwiching HDR surface mould brachytherapy using catheter flap between EBRT is a relatively safe and practical approach for post-mastectomy patients, with reasonable local control.

Treatment planning system (TPS) used in the TG-43 formalism-based on sources within an infinite water medium, without accounting for the scatter defect due to surrounding air [9]. The uncertainties related to the HDR brachytherapy dose calculation model (TG-43) should be acknowledged if HDR brachytherapy is used. The use of Acuros ${ }^{\circledR}$ BV dose calculation model would reduce this uncertainty. The presence of additional
B Comparison of OARs mean doses with TG-43 and Acuros ${ }^{\circledR}$ BV algorithm

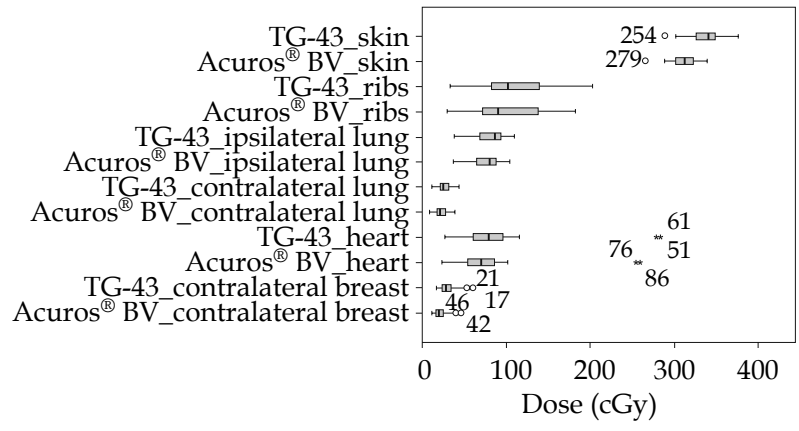

D Skin $\mathrm{V}_{150 \%}$ and $\mathrm{V}_{200 \%}$ volume comparison between TG-43 and Acuros ${ }^{\circledR}$ BV algorithm

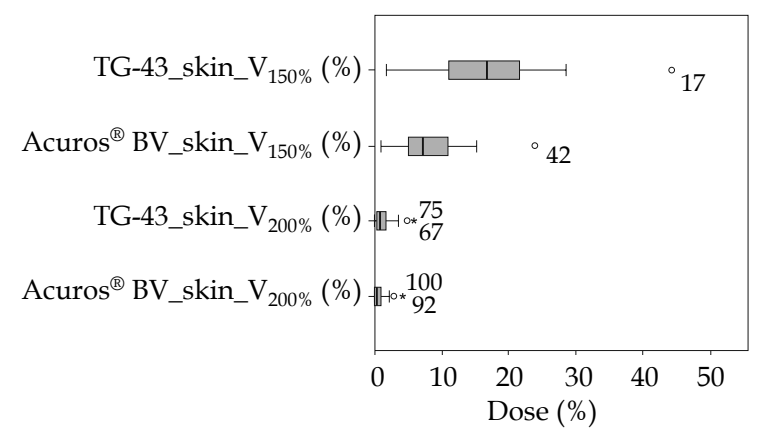

air gaps due to the moulds positional variation (from planned) would lead to underdosing the target volume, as the distance from the source positions is increased compared to the planned positions. In our simulations using Acuros ${ }^{\circledR}$ BV dose calculations, the addition of a 4- or 10-mm air gap between the mould and the skin decreased the dose by brachytherapy [12]. These two valid deficiencies and drawbacks were addressed in this study; firstly, by incorporating a thermoplastic mask over the surface mould to eliminate the air gap between the mould and the skin surface, helping in reproducibility within acceptable uncertainties with adequate coverage of prescription dose to the target volume, as shown in Figure 4. Secondly, taking heterogeneity algorithm Acuros ${ }^{\circledR}$ BV for dose computation accounts for actual scatter condition and the surrounding environment. Both Acuros ${ }^{\circledR}$ BV and advanced collapsed cone engine (Elekta, Stockholm, Sweden) have been shown to agree within $2 \%$ with Monte Carlo method calculations for single-source models [13], and specifically near the skin for breast brachytherapy patients' models [14]. The dose difference at the prescription depth ( $1 \mathrm{~cm}$ below the central catheter) increased with the growing treatment area. The dose overestimation of the TG-43 model decreased when a bolus was added above the treatment catheters [12]. This may not be a significant issue when heterogeneity algorithms are accepted in clinical use [15]. Comparison of GBBS and TG-43 for- 

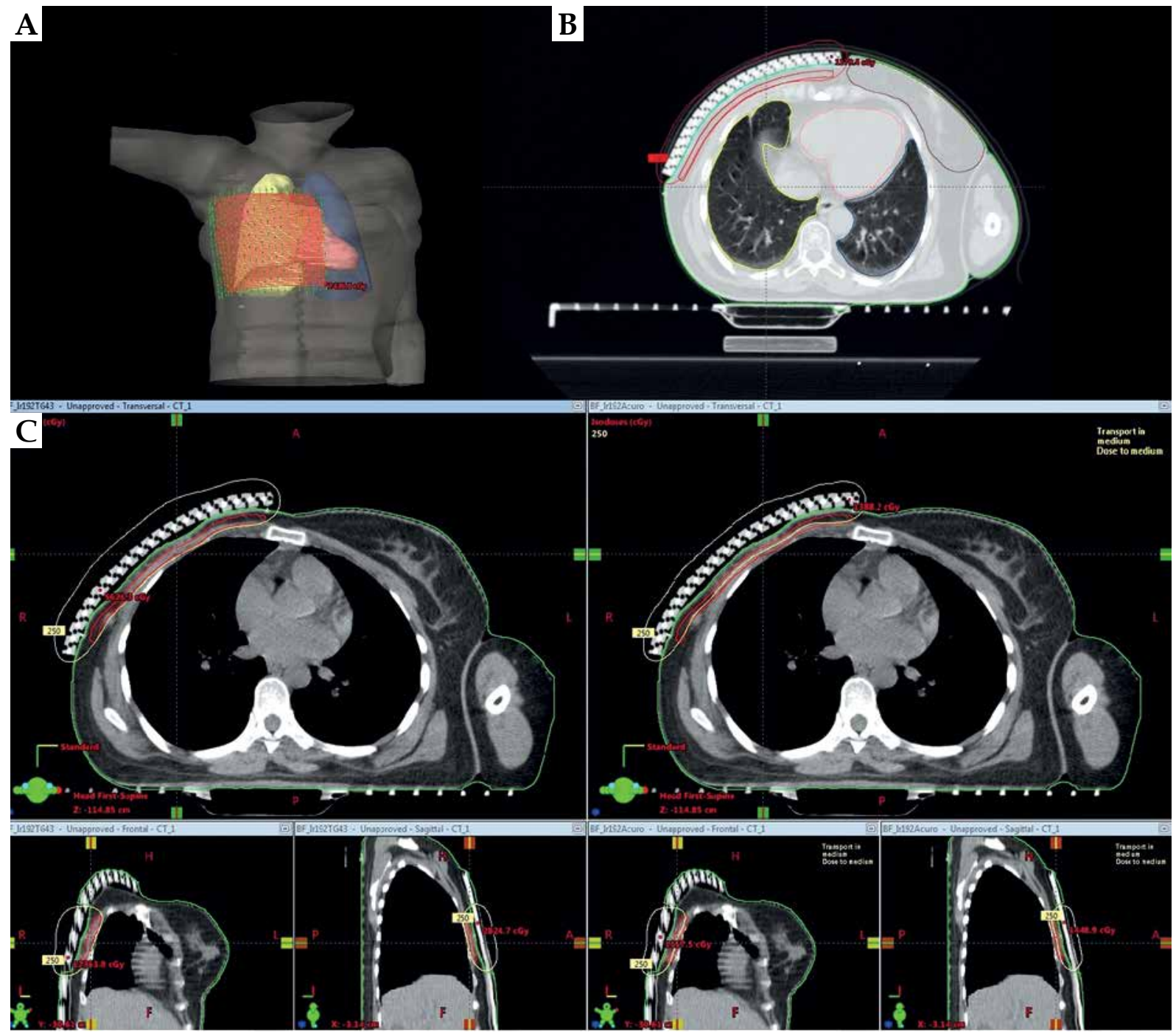

Fig. 4. A) Delineated organs at risk and clinical target volume with catheter reconstruction for scar boost irradiation. B) Clinical target volume enclosing the prescribed dose. C) The comparison of isodose distribution between TG-43 and Acuros ${ }^{\circledR}$ BV-based calculation in transverse, frontal, and sagittal planes

malism on breast interstitial metal catheters shows that the estimated dose to CTV was only marginally different from the two systems. There is a significant difference in estimated doses, ranging from $4 \%$ to $53 \%$ in the mean value of all parameters analyzed [16]. However, this uncertainty in HDR brachytherapy dose calculation is much more considerable than that observed in external beam dose calculations in the current clinical practice. Presently, the American Brachytherapy Society (ABS) working group [17] recommends using bolus as a backscatter material for skin HDR brachytherapy treatments.

In this study, CTV-HDR_evl received the prescribed dose as the mean value for $\mathrm{V}_{100 \%}$ of $70 \%(\mathrm{SD}=15.6)$ and their minimum dose was $67 \%(\mathrm{SD}=4.01)$ with Acuros $^{\circledR}$ BV-based planning; these doses were $25.6 \%$ and $10.9 \%$, respectively, lesser compared with TG-43-based planning. The dose coverage to $98 \%$ of the CTV-HDR_evl volume $\left(\mathrm{D}_{98 \%}\right)$, respectively, with their mean values of $86.19 \%$ (SD $=1.3)$, which were $10.4 \%$ reduced than TG-43-based dosimetry. These differences were noted without altering the dwell positions and achieved reduction due to actual scatter conditions. The mean dose (SD) to the heart was 0.91 Gy (12.4), which was $63.3 \%$ of the prescribed amount (PD) with TG-43-based calculation, and with Acuros ${ }^{\circledR}$ BV-based calculation it was $0.81 \mathrm{~Gy}$ (11.23), which was $67 \%$ of the prescribed dose (PD). A difference of $-3.7 \%$ was observed in the Acuros ${ }^{\circledR}$ BV calculation to the prescribed dose. These results are comparable with mean cardiac doses reported by a similar study ( $\mathrm{D}_{\text {mean }}$ of 0.9 Gy with TG-43 dosimetry) [7]. The dose receiving a $2.0 \%$ volume was 129.4 cGy with standard calculation and 122.02 cGy with Acuros ${ }^{\circledR}$ BV-based dosimetry, $-5.7 \%$ decrease was noted comparing with the current dosimetry technique.

The mean ipsilateral lung dose (MLD) was 76.49 cGy $(\mathrm{SD}=3.25$, range, 36.6-96.3 cGy) with heterogeneity algorithm, which was $-5.7 \%$ lesser compared with the TG-43 algorithm. $\mathrm{D}_{\max }$ and $\mathrm{D}_{2}$ \% values were $214.5 \mathrm{cGy}$ $(\mathrm{SD}=6.3$, range, 119.9-259.8 cGy) and $176.7 \mathrm{cGy}(\mathrm{SD}=5.18$, range, 94.41-208.8 cGy), which were about $85.8 \%$ and 
$70.6 \%$ of the PD, respectively. These values were $-10.9 \%$ and $-5.8 \%$ reduced compared to TG-43 formalism-based calculation. However, combining EBRT and HDR surface mould brachytherapy using catheter flap doses when added, the lung tolerance doses were within the tolerance value of $\mathrm{V}_{30 \mathrm{~Gy}}<20 \%$ and $\mathrm{V}_{20 \mathrm{~Gy}}<30 \%$, respectively. The mean dose to the skin was 311.04 cGy $(S D=3.33$, range, 265.7-339.8 cGy), and the volume receiving 150\% and $200 \%$ doses were received by $8.39 \%$ (SD $=0.99$, range $0.96-23.89 \%)$ with TG-43 algorithm and $0.76 \%(S D=0.20$, range $0.01-4.22 \%$ ) with Acuros ${ }^{\circledR} \mathrm{BV}$ algorithm. These doses were $-7.4 \%,-50 \%$, and $-39 \%$ lesser compared to TG-43-based calculations, and the values were significant. Higher doses to the skin were in expected lines that alter the scar boost dose from conventional $1000 \mathrm{cGy}$ to 750 cGy.

It is shown that the HDR surface mould brachytherapy with EBRT was a feasible alternative to EBRT alone in the treatment of chest wall irradiation. Our results demonstrate that OARs doses were lower with Acuros ${ }^{\circledR}$ $\mathrm{BV}$, which accurately considers the tissue inhomogeneities and scatter conditions. We have previously demonstrated that the dose difference between TG- 43 and Acu$\operatorname{ros}^{\circledR}$ BV calculations rises with increasing loading area for HDR brachytherapy surface mould treatments, resulting in a lower actual delivered dose than that seen with TG-43 dose calculation model [13]. The expected benefit of Acuros ${ }^{\circledR} \mathrm{BV}$ in dosimetry planning is in the amount of reduction it would achieve (through the individualization of patient's dosimetry) in a variance of response of clinical trial population [18].

\section{Conclusions}

Surface mould brachytherapy for scar boost irradiation combined with EBRT provides many advantages, particularly an alternative to electron beam therapy, and results are comparable in terms of local control rates, with a marginal increase in acute skin reactions. It also helps in reducing treatment time and treatment reproducibility. Large, irregular, and curved areas can be treated. The data comparing TG-43 formalism and Acuros ${ }^{\circledR}$ BV algorithms calculation in scar boost irradiation of breast are limited. This study shows how alternate planning algorithms account for the mass density of the medium, scatter condition, and actual dose prediction in a medium. The study also presented a reduced dose to associated organs and structures, which provides to report the correct dose delivered.

\section{Ethical consideration}

This study involves human and was performed in accordance with ethical standards. All the participants gave their written consent prior to the study. The study has been reviewed by an institute's ethics committee and was approved with reference No. JIP/IEC/1/2012/41.

\section{Disclosure}

The authors report no conflict of interest.

\section{References}

1. Horst KC, Haffty BG, Harris EE. ACR Appropriateness Criteria $^{\circledR}$ : postmastectomy radiotherapy. American College of Radiology, Reston, VA, USA 2012: 1-8.

2. Ma J, Li J, Xie J et al. Post mastectomy linac IMRT irradiation of chest wall and regional nodes: dosimetry data and acute toxicities. Radiat Oncol 2013; 8: 81.

3. Rong Y, Yadav P, Welsh JS et al. Postmastectomy radiotherapy with integrated scar boost using helical tomotherapy. Med Dosim 2012; 37: 233-239.

4. Mendenhall WM, Mancuso AA, Kirwan JM et al. Skin. In: Perez and Brady's principles and practice of radiation oncology. Wolters Kluwer Health, New York 2018.

5. Stewart AJ, O'Farrell DA, Bellon JR et al. CT computer-optimized high-dose-rate brachytherapy with surface applicator technique for scar boost radiation after breast reconstruction surgery. Brachytherapy 2005; 4: 224-229.

6. Frakulli R, Galuppi A, Cammelli $S$ et al. Brachytherapy in non melanoma skin cancer of eyelid: a systematic review. J Contemp Brachytherapy 2015; 7: 497-502.

7. Vijayaprabhu N, Gunaseelan K, Vivekanandan N et al. Postmastectomy scar boost irradiation using HDR surface mould brachytherapy by 3D image-based volume optimization. Int I Med Phys Clin Engineering Radiat Oncol 2013; 2: 139.

8. Taylor ME, Haffty BG, Rabinovitch R et al. ACR Appropriateness Criteria ${ }^{\circledR}$ on postmastectomy radiotherapy: expert panel on radiation oncology - breast. Int J Radiat Oncol Biol Phys 2009; 73: 997-1002.

9. Granero D, Perez-Calatayud J, Vijande J et al. Limitations of the TG-43 formalism for skin high-dose-rate brachytherapy dose calculations. Med Phys 2014; 41: 021703.

10. Chagpar A, Kuerer HM, Hunt KK et al. Outcome of treatment for breast cancer patients with chest wall recurrence according to initial stage: implications for post-mastectomy radiation therapy. Int J Radiat Oncol Biol Phys 2003; 57: 128-135.

11. Youssef A, Xue J, Chen $Y$ et al. Chest wall scar boost using high-dose-rate freiberg flap and comparison to electron field. Brachytherapy 2014; 13: S84-85.

12. Boman EL, Satherley TW, Schleich N et al. The validity of Acuros BV and TG-43 for high-dose-rate brachytherapy superficial mould treatments. Brachytherapy 2017; 16: 1280-1288.

13. Zourari K, Pantelis E, Moutsatsos A et al. Dosimetric accuracy of a deterministic radiation transport based brachytherapy treatment planning system. Part I: Single sources and bounded homogeneous geometries. Med Phys 2010; 37: 649-661.

14. Zourari K, Pantelis E, Moutsatsos A et al. Dosimetric accuracy of a deterministic radiation transport based 192Ir brachytherapy treatment planning system. Part III. Comparison to Monte Carlo simulation in voxelized anatomical computational models. Med Phys 2013; 40: 011712.

15. Beaulieu L, Carlsson Tedgren $\AA$, Carrier JF et al. Report of the Task Group 186 on model-based dose calculation methods in brachytherapy beyond the TG-43 formalism: current status and recommendations for clinical implementation. Med Phys 2012; 39: 6208-6236.

16. Sinnatamby M, Nagarajan V, Reddy KS et al. Dosimetric comparison of Acuros ${ }^{\mathrm{TM}}$ BV with AAPM TG43 dose calculation formalism in breast interstitial high-dose-rate brachytherapy with the use of metal catheters. J Contemp Brachytherapy 2015; 7: 273-279.

17. Ouhib Z, Kasper M, Calatayud JP et al. Aspects of dosimetry and clinical practice of skin brachytherapy: The American Brachytherapy Society working group report. Brachytherapy 2015; 14: 840-858.

18. Papagiannis P, Pantelis E, Karaiskos P. Current state of the art brachytherapy treatment planning dosimetry algorithms. Br J Radiol 2014; 87: 2014016. 